\title{
One-electron excitations, correlation effects, and the plasmon in cesium metal
}

\author{
Andrzej Fleszar \\ Physikalisches Institut, Universität Würzburg, Am Hubland, 97074 Würzburg, Germany; \\ Department of Physics and Astronomy, The University of Tennessee, Knoxville, Tennessee 37996-1200; \\ and Solid State Division, Oak Ridge National Laboratory, Oak Ridge, Tennessee 37831-6032 \\ Roland Stumpf \\ Sandia National Laboratories, Division 1114, Albuquerque, New Mexico 87185-0344 \\ Adolfo G. Eguiluz \\ Fritz-Haber-Institut der Max-Planck-Gesellschaft, Faradayweg 4-6, D-14195 Berlin, Germany; \\ Department of Physics and Astronomy, The University of Tennessee, Knoxville, Tennessee 37996-1200; \\ and Solid State Division, Oak Ridge National Laboratory, Oak Ridge, Tennessee 37831-6032
}

(Received 9 October 1996)

\begin{abstract}
We study the dynamical electronic response of $\mathrm{Cs}$ at a first-principles level. The spatially localized $5 p$ semicore shell induces a physical interplay between crystal local fields and electron correlations, leading to a novel, and relatively large, many-body shift of the plasmon energy. This effect, combined with that of oneelectron transitions into empty states near the plasmon energy, yields a plasmon dispersion curve which is in excellent agreement with experiment for small wave vectors. In addition, our results feature a flat dispersion for large wave vectors, in qualitative agreement with experiment. [S0163-1829(97)07504-8]
\end{abstract}

The physics of alkali metals has attracted renewed attention in recent years, as several experiments reported findings which were in conflict with the belief that these metals were well understood. ${ }^{1-5}$ Particularly noteworthy is the fact that the experimentally determined ${ }^{5}$ plasmon dispersion relation of the heavy alkali metals ( $\mathrm{Rb}$ and $\mathrm{Cs}$ ) is in complete disagreement with the textbook behavior expected for a "simple" metal. ${ }^{6}$ The high-resolution electron energy loss spectroscopy (EELS) experiments of vom Felde, SprösserProu, and Fink ${ }^{5}$ revealed that, in the case of $\mathrm{Cs}$, the dispersion of the plasmon energy for small wave vectors is negative. Moreover, for large wave vectors the dispersion relation is quite flat, in marked contrast with the strong dispersion predicted by available electron-liquid theories for electrons in jellium with the bulk density of $\mathrm{Cs}, r_{S}=5.6$. This situation led to the tantalizing conjecture that the physics of $\mathrm{Cs}$, which has the lowest valence-electron density of all elemental metals, is dominated by strong electron-electron correlations 5 - even the possibility of an incipient Wigner crystal state has been raised.

Very recently, Aryasetiawan and Karlsson ${ }^{7}$ reported $a b$ initio calculations of the plasmon dispersion relation for the alkali metals, performed within the random phase approximation (RPA). ${ }^{6}$ In the case of Cs, these authors obtained a negative dispersion for small wave vectors, a result which was traced to the effects of the band structure.

The issue of the role played by correlation for electrons propagating in the actual band structure of Cs has not been settled thus far. Phenomenological arguments have been put forth suggesting that the many-body effects strongly influence the dispersion of the Cs plasmon. ${ }^{8}$ An opposite viewpoint was held in Ref. 7. However, the value of the plasmon energy $\left(\omega_{p}\right)$ obtained by Aryasetiawan and Karlsson ${ }^{7}$ is too high; it is about as high as that for jellium. Since the all- electron calculations of Ref. 7 include contributions from both valence and core states, this feature must be a signature of the physics left out by RPA response, namely correlation. This question, together with the "unwritten theorem" that charge localization-such as introduced by the large Cs core-enhances the many-body correlations, ${ }^{9}$ calls for an investigation of these effects for real Cs.

In this paper we show that the electron-electron correlations enter the plasmon dispersion relation of $\mathrm{Cs}$ in a nontrivial way. The spatially localized $5 p$ semicore enhances the crystal local fields, and these in turn magnify the impact of the correlations, which in effect renormalize $\omega_{p}$. Thus, while one-electron transitions into final states lying just above $\omega_{p}$ are the root of the "anomalous" negative plasmon dispersion, ${ }^{5}$ the correlation effect, which we treat via a vertex correction, must be accounted for in order to reproduce the measured dispersion relation for small wave vectors. ${ }^{10}$

The theoretical scheme used in the present work is similar to the one we implemented recently in an ab initio study of the inelastic $\mathrm{x}$-ray scattering spectrum of Al. ${ }^{11}$ The simplest approximation for the irreducible polarizability $\widetilde{\chi}\left(\mathbf{x}, \mathbf{x}^{\prime} ; \omega\right)$ is given by the RPA bubble $\chi^{(0)}\left(\mathbf{x}, \mathbf{x}^{\prime} ; \omega\right)$, for which we have the well-known Fourier representation

$$
\begin{aligned}
\chi_{\mathbf{G}, \mathbf{G}^{\prime}}^{(0)}(\mathbf{q} ; \omega)= & \frac{1}{\Omega_{N}} \sum_{\mathbf{k}}^{\mathrm{BZ}} \sum_{n, n^{\prime}} \frac{f_{\mathbf{k}, n}-f_{\mathbf{k}+\mathbf{q}, n^{\prime}}}{E_{\mathbf{k}, n}-E_{\mathbf{k}+\mathbf{q}, n^{\prime}}+\hbar(\omega+i \eta)} \\
& \times\left\langle\mathbf{k}, n\left|e^{-i(\mathbf{q}+\mathbf{G}) \cdot \hat{\mathbf{x}}}\right| \mathbf{k}+\mathbf{q}, n^{\prime}\right\rangle \\
& \times\left\langle\mathbf{k}+\mathbf{q}, n^{\prime}\left|e^{i\left(\mathbf{q}+\mathbf{G}^{\prime}\right) \cdot \hat{\mathbf{x}}}\right| \mathbf{k}, n\right\rangle,
\end{aligned}
$$

where $\Omega_{N}$ is the normalization volume and $\mathbf{G}$ is a vector of the reciprocal lattice. The matrix elements in Eq. (1) were evaluated by expanding the one-electron Bloch states in a 


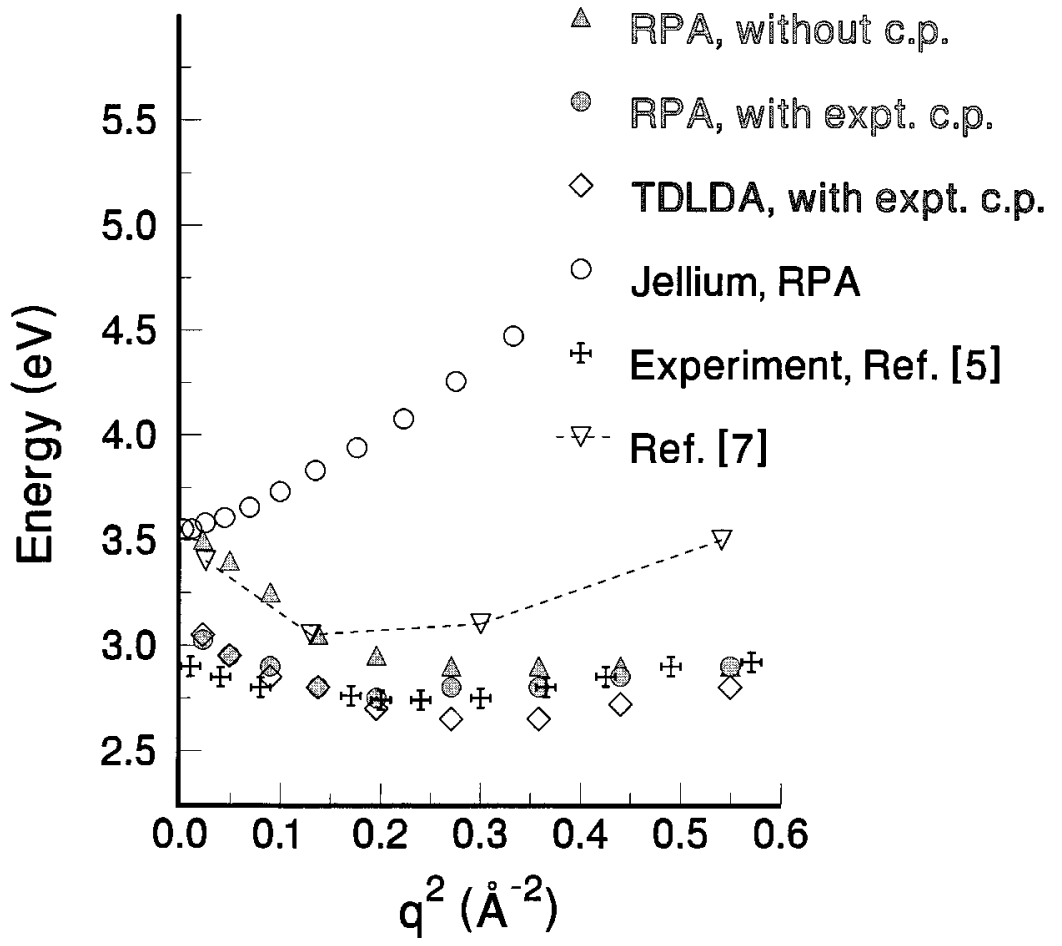

FIG. 1. Plasmon dispersion relation in Cs. Theoretical curves are for propagation along the (110) direction, and refer to calculations in which the $5 p$ states are assigned to the core. Results "with (without) expt. c.p." include (do not include) the effect of the experimentally measured core polarizability (see text).

plane-wave basis. The coefficients of such an expansion were obtained from the self-consistent solution of the ground state in the local-density approximation (LDA) of densityfunctional theory. ${ }^{12}$ In the computation of $\chi^{(0)}$ according to Eq. (1) typically we included 20 bands, sampled the Brillouin zone with use of 240 wave vectors in the irreducible element, and introduced a broadening parameter given by $\eta=0.1 \mathrm{eV}$.

Contact with the EELS experiments of Ref. 5 is made through the density-response function $\chi\left(\mathbf{x}, \mathbf{x}^{\prime} ; \omega\right){ }^{6}$ In particular, the energy of the plasmon for a given wave vector is obtained from the main peak in $\operatorname{Im} \chi_{\mathbf{G}=0, \mathbf{G}^{\prime}=0}(\mathbf{q} ; \omega)$, which is proportional to the dynamical structure factor $S(\mathbf{q} ; \omega) .{ }^{11} \mathrm{We}$ evaluate $\chi$ by solving the integral equation it satisfies, ${ }^{13}$ $\chi=\tilde{\chi}+\tilde{\chi} \nu \chi$ (written in symbolic form), where $\nu$ is the bare Coulomb interaction. In the present work many-body effects are introduced via a "many-body local field" $G(\mathbf{q}),{ }^{13-15}$ or "vertex correction" $f_{\mathrm{xc}}=-\nu G$, in terms of which

$$
\tilde{\chi}^{-1}=\left(\chi^{(0)}\right)^{-1}-f_{\mathrm{xc}} .
$$

The structure of Eq. (2) corresponds to an approximate summation of the exchange ladders, ${ }^{15}$ and leads to a response function of the form

$$
\chi=\chi^{(0)}\left[1-\nu(1-G) \chi^{(0)}\right]^{-1} .
$$

Equation (2) arises rigorously-with an $\omega$-dependent vertex-in recent developments of time-dependent densityfunctional theory, where $f_{\mathrm{xc}}$ is defined as the functional derivative with respect to the density of the time-dependent exchange-correlation potential. ${ }^{16}$ However, the precise functional form of this potential is not known; thus, current applications of the density-functional method typically resort to making an adiabatic ansatz, such as the popular "timedependent', LDA (TDLDA), ${ }^{17}$ in which $f_{\mathrm{xc}}$ $=\int d^{3} x e^{-i \mathbf{q} \cdot \mathbf{x}} d V_{\mathrm{xc}}(\mathbf{x}) / d n(\mathbf{x}), \quad$ where $\quad V_{\mathrm{xc}}(n)$ is the exchange-correlation potential obtained in an LDA groundstate calculation. An interesting feature of our findings is that the crystal local fields induced by the Cs core actually probe the nonlocality of the vertex.

For clarity in the presentation we discuss our results in two stages. The first stage (Figs. 1 and 2) starts out from an LDA band structure constructed with use of an $a b$ initio pseudopotential $^{18}$ in which the $5 p$ shell is assigned to the core ${ }^{19} \mathrm{cf}$. the $\mathrm{Xe} 6 s$ configuration of $\mathrm{Cs}$ in the Periodic Table. In Fig. 1 we present results for plasmon propagation along the (110) direction. As a useful benchmark, the figure shows the dispersion relation for jellium $\left(r_{S}=5.6\right)$, obtained in the RPA; the same is clearly in qualitative disagreement with experiment ${ }^{5}$-it was precisely this situation which prompted the conclusion that in Cs there may be a fundamental breakdown of the existing theories of the response of the interacting electron liquid. ${ }^{5}$ Two of our RPA dispersion curves $\left(f_{\mathrm{xc}}=0\right)$ for band electrons are shown in Fig. 1; both are endowed with the "anomalous" features of the experimental data, ${ }^{5}$ namely negative dispersion for small $q$ 's and flat dispersion for large $q$ 's. Now the dispersion curve which ignores the polarizability of the core is manifestly inaccurate for $q \rightarrow 0$. Interestingly, the RPA result of Ref. 7 (also shown in Fig. 1) has a similar $q \rightarrow 0$ limit, despite the all-electron nature of that work; we attribute this shortcoming to the neglect of correlation effects-see below. The dispersion curve we obtained upon adding to the real part of the dielectric function of $\mathrm{Cs}$ the experimental value of the core polarizability ${ }^{20}$ agrees with the data of vom Felde et al. ${ }^{5}$ very well, for all $q$ 's.

Now, the EELS experiments of vom Felde et al. ${ }^{5}$ were performed on polycrystalline samples. Calculations for other directions result in dispersion curves which depend rather sensitively on propagation direction for large $q$ 's. ${ }^{10,21}$ Furthermore, many-body corrections to the RPA tend to lower 

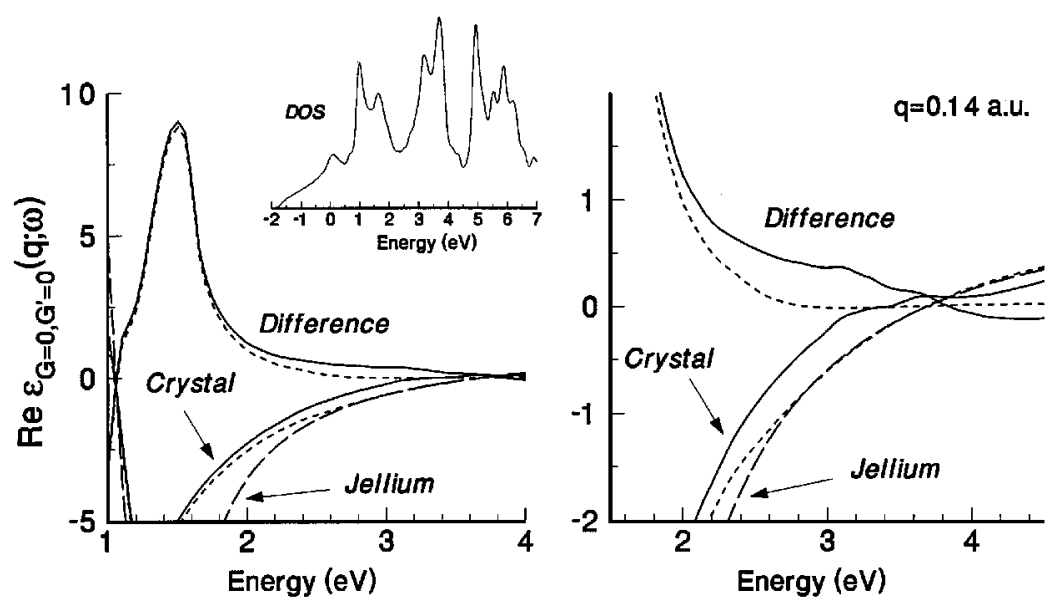

FIG. 2. $\operatorname{Re} \varepsilon_{\mathbf{G}=0, \mathbf{G}^{\prime}=0}(\mathbf{q} ; \omega)$ for Cs, for its jellium counterpart, and their difference, calculated in the RPA. The wave vector $\mathbf{q}$ is along the (110) direction $(|\mathbf{q}|=0.14$ a.u.). Dashed curves are explained in the text. The right panel is a blowup of the left panel in the vicinity of the root of $\operatorname{Re} \varepsilon_{\mathbf{G}=0, \mathbf{G}^{\prime}=0}(\mathbf{q} ; \omega)=0$. The inset shows the calculated density of one-electron states (the $5 p$ states are assigned to the core).

the dispersion curve for large $q$ 's, as illustrated by the TDLDA result given in Fig. 1. For all of these reasons we feel that the quantitative agreement between our RPA results of Fig. 1 and the data of Ref. 5 for large q's is less significant than the fact that our results are nonjelliumlike, i.e., the plasmon is rather dispersionless, for large wave vectors. It would be very interesting for EELS experiments to be performed on a Cs single crystal; this would help clarify the band-structure effects behind the anisotropies present in our calculated spectra $^{20,21}$ and also the nature of the many-body correlations for large $q$ 's.

The drastic difference between the plasmon dispersion curves for band electrons and for electrons in jellium is explained in Fig. 2, which centers on the effect of the unoccupied band structure on the position of the root of $\operatorname{Re} \varepsilon_{\mathbf{G}=0, \mathbf{G}^{\prime}=0}(\mathbf{q} ; \omega)=0$-an alternative definition of the energy of the plasmon for a given $\mathbf{q}$. The prominent spike due to one-electron transitions into a $p d$ complex $^{22}$ lying at $\sim 1-2 \mathrm{eV}$ above $E_{F}$ (see inset) is clearly the leading spectral feature. It may then seem that the anomalous dispersion obtained in Fig. 1 is due to those transitions, which would make the present problem akin to the classic case of the $3.8 \mathrm{eV}$ plasmon of Ag. However, this is not the case. The short dashes in Fig. 2 show the result we obtained upon eliminating from Eq. (1) all states lying higher than $2.5 \mathrm{eV}$ above $E_{F}$; it is apparent that, in the absence of those transitions, which are dominated by a $d$ complex ${ }^{22}$ lying at $3-4 \mathrm{eV}$ above $E_{F}$ (see inset), the root of $\operatorname{Re} \varepsilon_{\mathbf{G}=0, \mathbf{G}^{\prime}=0}(\mathbf{q} ; \omega)=0$ is the same as in the case of jellium. The fact that this band complex lies so close to $\omega_{p}$ explains its importance, the small spectral weight of the corresponding transitions in Fig. 2 notwithstanding. Finally, we note that the intensity of these transitions grows with $q$ (not shown here for brevity), and this enhances the downward shift of the energy of the plasmon relative to its value for jellium-hence the "anomalous" dispersion obtained in Fig. 1.

Now, the role played by the Cs core in Fig. 1 was simply to provide a polarizable background which lowered $\omega_{p}$ by $\sim 0.5 \mathrm{eV} .^{23}$ However, the collective response of Cs bears a more fundamental imprint of the presence of the polarizable core, a consequence of the dual enhancement of many-body correlations and crystal local fields to which it gives rise.

This finding is addressed in the "second stage" of our calculations, which start out from the construction of an $a b$ initio pseudopotential in which the $5 p$ orbitals are treated as valence states. (The new pseudopotential was produced for the ionic configuration $5 p 6,6 s 0.7$; we did not introduce an additional projector for the $6 p$ states. $^{24}$ ) This allows us to treat the semicore states on the same footing with the "conventional" valence states in the evaluation of $\chi^{(0)}$ according to Eq. (1). The main modification required in our procedure to handle these rather localized states is the use of a larger number of plane waves: the kinetic energy cutoff is now 15 Ry, while in Figs. 1 and 2 we used 8 Ry.

The left panel of Fig. 3 shows the plasmon dispersion curve obtained from a scalar version of Eq. (3), in which $\chi_{\mathbf{G}=0, \mathbf{G}^{\prime}=0}$ is evaluated from the knowledge of just the $\mathbf{G}=\mathbf{G}^{\prime}=0$ element of $\chi^{(0)}$. Such solution is said to ignore the
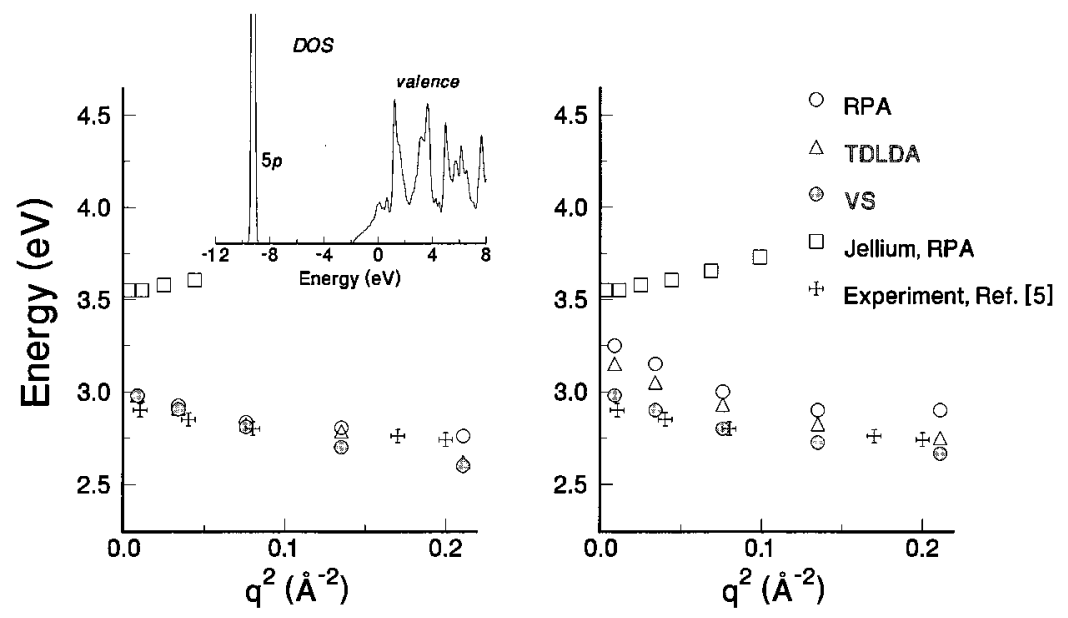

FIG. 3. Plasmon dispersion relation in Cs for small wave vectors along the (110) direction. The $5 p$ semicore states are treated as valence states. Right (left) panel includes (ignores) the crystal local fields. Theoretical curves are labeled by the vertex correction used in the respective calculation; see text. Inset: calculated density of oneelectron states. 
"crystal local fields,", i.e., the contribution to the screening from density fluctuations of wavelengths comparable with the lattice constant. Dispersion curves computed in the presence of the crystal local fields are shown on the right panel. Both panels include three sets of calculations, each set referring to a different approximation for the electron-electron correlations. These are, respectively, the RPA, which ignores correlation altogether, the TDLDA, whose $f_{\mathrm{xc}}$ was defined below Eq. (3), and Vashishta-Singwi (VS). ${ }^{14}$ The comparison between the two panels is quite striking, as it reveals the presence of a sizable many-body effect for small wave vectors.

We explain this result in two steps. First we note that the full calculation proceeds via the evaluation of the polarizability matrix $\chi_{\mathbf{G}, \mathbf{G}^{\prime}}^{(0)}$, with a subsequent inversion of the matrix $\left[1-\nu(1-G) \chi^{(0)}\right]^{-1}$ to yield the response $\chi_{\mathbf{G}, \mathbf{G}^{\prime}}$, whose $\mathbf{G}=\mathbf{G}^{\prime}=0$ element gives the loss spectrum $S(\mathbf{q} ; \omega) .{ }^{11}$ Recalling the labeling convention $\chi_{\mathbf{G}, \mathbf{G}^{\prime}}(\mathbf{q} ; \omega) \equiv \chi(\mathbf{q}+\mathbf{G}, \mathbf{q}$ $\left.+\mathbf{G}^{\prime} ; \omega\right)$ we recognize that, through the process of matrix inversion, there is a "feedback" between large wave-vector arguments $\mathbf{q}+\mathbf{G}$ in $\chi^{(0)}$ and the small wave vector $\mathbf{q}$ of the plasmon. Now, the crystal local fields by themselves shift the energy of the plasmon upwards, as can be seen by comparing the RPA results in both panels of Fig. 3. This is a kineticenergy (or electron-gas pressure) effect. That the shift is towards higher energies can be understood by analogy with the positive dispersion of the uniform electron-gas plasmon as $q$ grows from zero.

The introduction of a vertex correction in Eq. (3), in conjunction with this "mixing" of large and small wave vectors, induces a competing effect, i.e., a downward shift of $\omega_{p}$. This effect is consistent with the physical interpretation of the many-body local field factor $G(q)$ - the same accounts for the presence of an exchange-correlation hole about each screening electron, which clearly weakens the screening. From the numerical viewpoint we have that the factor $[1-G(\mathbf{q})]$ tends to screen out the Coulomb interaction in Eq. (3) for typical arguments $\mathbf{q}+\mathbf{G}$ entering the matrix to be inverted, and this weakens the "restoring force" responsible for the collective motion-thus the many-body shift to lower energies.

It is important to note that what is new here is that the above effect occurs for small wave vectors, and this is so because of the intrinsic matrix nature of the response in the presence of the $5 p$ semicore. Indeed, for a scalar response the effect vanishes, as $G(q) \rightarrow 0$ for $q \rightarrow 0$. We stress that when the $5 p$ states were kept in the core (Fig. 1) we obtained identical results whether Eq. (3) was solved as a matrix equation or not. In other words, the presence of the semicore enhances the crystal local fields, and with them an interplay sets in between one-electron excitations for the large wave vectors contained in $\chi^{(0)}$ and the plasmon response for small wave vectors.

We note that the VS vertex used above is one of the many corrections to the RPA introduced over the years for the homogeneous electron gas. This particular vertex function has the appeal of its intuitive physical derivation; ${ }^{13,14}$ furthermore, it has been parametrized as a simple function of $q$, which makes it easy to use. The important "generic' feature of the VS $f_{\mathrm{xc}}(\mathbf{q})$ is its realistic wave-vector dependence; other approximations are expected to yield similar results. ${ }^{25}$ By contrast, the local approximation renders the TDLDA vertex inherently $q$-independent in the absence of crystallinity. The results of Fig. 3 clearly improve with the inclusion of nonlocality in the vertex.

In conclusion, we have shown that the measured dispersion relation of the Cs plasmon ${ }^{5}$ contains a built-in signature of the many-body correlations between the electrons. These correlations, together with the crystal local fields, are enhanced by the spatially localized core. A rather simple description of the many-body effect, combined with an accurate treatment of the one-electron transitions near $\omega_{p}$, yields quantitative agreement with experiment for small wave vectors. The physics discussed in this paper should be of relevance for a variety of systems, particularly those with extended semicore states. $^{26}$

We thank J. A. Gaspar for helpful discussions during the course of this work. A.G.E. acknowledges support from NSF Grant No. DMR-9207747/9596022, the San Diego Supercomputer Center, and the National Energy Research Supercomputer Center. He also acknowledges the support of the Alexander von Humboldt Stiftung through a U.S. Senior Scientist Award. Oak Ridge National Laboratory is managed by Lockheed Martin Energy Systems, Inc., for the Division of Materials Sciences, U.S. DOE under Contract No. DEAC05-84OR21400.
${ }^{1}$ E. Jensen and E. W. Plummer, Phys. Rev. Lett. 55, 1912 (1985); P. H. Citrin et al., ibid. 61, 1021 (1988); B. S. Itchkawitz et al., ibid. 68, 2488 (1993).

${ }^{2}$ K. W.-K. Shung and G. D. Mahan, Phys. Rev. Lett. 57, 1076 (1986); Phys. Rev. B 38, 3856 (1988); J. E. Northrup, M. S. Hybertsen, and S. G. Louie, Phys. Rev. Lett. 59, 819 (1987); Phys. Rev B 39, 8198 (1989).

${ }^{3}$ X. Zhu and A. W. Overhauser, Phys. Rev. B 33, 925 (1986); A. W. Overhauser, Phys. Rev. Lett. 55, 1916 (1985).

${ }^{4}$ S.-K. Ma and K. W.-K. Shung, Phys. Rev. B 49, 10617 (1994).

${ }^{5}$ A. vom Felde, J. Sprösser-Prou, and J. Fink, Phys. Rev. B 40, 10181 (1989); A. vom Felde et al., Europhys. Lett. 4, 1037 (1987)

${ }^{6}$ D. Pines and P. Nozières, The Theory of Quantum Liquids (Ben- jamin, New York, 1966), Vol. 1.

${ }^{7}$ F. Aryasetiawan and K. Karlsson, Phys. Rev. Lett. 73, 1679 (1994).

${ }^{8}$ M. Taut, J. Phys. Condens. Matter 4, 9595 (1992); M. Taut and K. Sturm, Solid State Commun. 82, 295 (1992).

${ }^{9}$ A. Muramatsu and W. Hanke, Phys. Rep. 113, 97 (1984).

${ }^{10} \mathrm{~A}$ preliminary account of an early stage of this work has been given elsewhere; A. G. Eguiluz, A. Fleszar, and J. Gaspar, Nucl. Instrum. Methods B 96, 550 (1995).

${ }^{11}$ A. Fleszar, A. A. Quong, and A. G. Eguiluz, Phys. Rev. Lett. 74, 590 (1995)

${ }^{12}$ W. Kohn and L. J. Sham, Phys. Rev. 140, A1133 (1965).

${ }^{13}$ G. D. Mahan, Many-Particle Physics, 2nd ed. (Plenum, New York, 1990). 
${ }^{14}$ P. Vashishta and K. S. Singwi, Phys. Rev. B 6, 875 (1972).

${ }^{15}$ J. Hubbard, Proc. R. Soc. London, Ser. A 243, 336 (1957).

${ }^{16}$ E. K. U. Gross and W. Kohn, Adv. Quantum Chem. 21, 255 (1990); M. Petersilka and E. K. U. Gross, Phys. Rev. Lett. 76, 1212 (1996).

${ }^{17}$ A. Zangwill and P. Soven, Phys. Rev. A 21, 1561 (1980).

${ }^{18}$ D. R. Hamann, Phys. Rev. B 40, 2980 (1989).

${ }^{19}$ The nonlinear dependence of $V_{\mathrm{xc}}(n)$ on the total (core plus valence) electron density of the $\mathrm{Cs}$ atom is accounted for via a "partial-core correction," as discussed by S. G. Louie, S. Froyen, and M. L. Cohen, Phys. Rev. B 26, 1738 (1982).

${ }^{20}$ U. S. Whang, E. T. Arakawa, and T. A. Callcott, Phys. Rev. B 6, 2109 (1972).

${ }^{21}$ The dispersion curve for the (111) direction lies below that for the
(110) direction by $\sim 0.2 \mathrm{eV}$ for large $q$ 's; for the (100) direction, the "fine structure" in $S(\mathbf{q} ; \omega)$ becomes so prominent that it becomes difficult to identify the plasmon for $q \geqslant 0.4 \AA^{-1}$ (Ref. $10)$.

${ }^{22}$ D. A. Papaconstantopoulos, Handbook of the Band Structure of Elemental Solids (Plenum, New York, 1986).

${ }^{23}$ K. Sturm, E. Zaremba, and K. Nuroh, Phys. Rev. B 42, 6973 (1990), have reported calculations of the effect of the core on the dielectric properties of simple metals. The effects discussed in the present paper are not contained in that work.

${ }^{24}$ P. E. Blöchl, Phys. Rev. B 41, 5414 (1990).

${ }^{25}$ S. Moroni, D. M. Ceperley, and G. Senatore, Phys. Rev. Lett. 75, 689 (1995).

${ }^{26}$ D. Singh, Phys. Rev. B 43, 6388 (1991). 